\title{
Primordial Hematopoietic Stem Cells Generate Microglia But Not Myelin-Forming Cells in a Neural Environment
}

\author{
Sandrine Vitry, ${ }^{1}$ Julien Y. Bertrand, ${ }^{2}$ Ana Cumano, ${ }^{2}$ and Monique Dubois-Dalcq ${ }^{1}$ \\ ${ }^{1}$ Unité de Neurovirologie et Régénération du Système Nerveux and ${ }^{2}$ Unité du Développement des Lymphocytes, Unité de Recherche Associée, Centre \\ National de la Recherche Scientifique 1961, Institut Pasteur, 75724, Paris, Cedex 15, France
}

Finding ways to enhance remyelination is a major challenge in treating demyelinating diseases. Recent studies have suggested that circulating bone marrow cells can home in brain and transdifferentiate into neural cells. To ask whether hematopoietic precursors can form myelinating cells, we investigated the neuropoietic potential of embryonic precursors sorted from the mouse aorta-gonadsmesonephros (AGM) region. This cell fraction is capable of long-term hematopoietic reconstitution and generates colonies containing multipotential precursors and lymphoid or erythro-myeloid progenies. When cultured in hematopoietic growth conditions, a fraction of CD45-positive AGM cells coexpress neural markers such as nestin, the polysialylated form of neural cell adhesion molecule, the $\beta$ III tubulin isoform, and glial fibrillary acidic protein. However, when hematopoietic precursors containing green fluorescent protein were cocultured with embryonic striatal precursors into neurospheres, they maintained their hematopoietic phenotype without undergoing differentiation into neurons, astrocytes, or oligodendrocytes. After intraventricular grafting, hematopoietic precursors integrated into the brain of wild-type or hypomyelinated newborn shiverer mice and gave rise to microglia but not neurons or glia. In contrast, when wild-type embryonic striatal neurospheres were grafted in shiverer, they formed numerous myelin internode patches. Even when neural and hematopoietic precursors were grafted together into shiverer mice, only neural precursors generated myelin-forming cells and synthesized myelin. Thus, embryonic neurospheres have myelin repair properties not shown by embryonic hematopoietic precursors. This suggests that the use of multipotential neural precursors to generate myelin-forming cells remains one of the most promising avenues toward remyelination therapies.

Key words: neural stem cells; oligodendrocytes; shiverer; hematopoietic stem cells; microglia; myelin repair

\section{Introduction}

Hematopoietic stem cells (HSCs) are well known to give rise to erythrocytes, myelocytes, and lymphocytes and neural stem cells to neurons, oligodendrocytes, and astrocytes. Recently, it was shown that stem cells of one type can also produce cells normally derived from the other, a property called "stem cell plasticity" (Temple, 2001). Indeed, grafted cells from brain-derived neurospheres were shown to home in bone marrow $(\mathrm{BM})$ and produce hematopoietic colonies in sublethally irradiated hosts (Bjornson et al., 1999). Stem cell plasticity was also invoked in studies showing that grafted BM-derived cells can traffic to brain and generate neural cells in the CNS of immunodeficient mice and men (Eglitis and Mezey, 1997; Brazelton et al., 2000; Mezey and Chandross, 2000; Mezey et al., 2003; Weimann et al., 2003). Because such plasticity holds considerable promise for therapy of neurodegen-

Received Aug. 25, 2003; revised Sept. 25, 2003; accepted Sept. 25, 2003.

This study was supported by a grant from the French Ministery of Research (A.C., M.D.D.). We thank Institut National de la Santé et de la Recherche Médicale, the Association pour la Recherche sur la Sclèrose en Plaques, and the European Community (Grant QLG3-CT-2000-00911) for their support (M.D.D., S.V.). The "Ligue contre le Cancer" funded the laboratory of A.C., which is associated with this foundation. We are very thankful to our colleagues for critical reading of this manuscript and discussion of the data: Heinz Arnheiter, Pascale Durbec, Isabelle Franceschini, and Jean-Michel Heard, as well as Kerren Murray and Doris Wang. The PSA and 04 antibodies were a gift from Genevieve Rougon and Sue Barnett, respectively.

Correspondence should be addressed to Monique Dubois-Dalcq, Unité de Neurovirologie et Régénération du Système Nerveux, Institut Pasteur, 25 rue du Dr. Roux, 75724, Paris, Cedex 15, France. E-mail: mdalcq@pasteur.fr. Copyright $\odot 2003$ Society for Neuroscience $\quad$ 0270-6474/03/2310724-08\$15.00/0 erative diseases, it is not surprising that it has generated both excitement and controversy (Weissman et al., 2001; Lemischka, 2002; Meletis and Frisen, 2003).

In the field of demyelinating diseases, BM transplantation (BMT) has been beneficial in X-linked adrenoleukodystrophy (Shapiro et al., 2000). In addition, adult BM cells, delivered in a focal demyelinated lesion or intravenously, induced remyelination in rat spinal cord (Sasaki et al., 2001; Akiyama et al., 2002a). Given the potential that BM cells can contribute to nervous tissue, it is of critical importance to carefully characterize the cell types involved and the conditions favoring cell fate switches. If the neurogenic cells in BM are indeed HSCs, we reasoned that the most immature HSCs should display the highest degree of plasticity. In mouse, primitive HSCs emerge in the aorta-gonads-mesonephros (AGM) region at embryonic day 10.5. These cells do not differentiate in situ and thus contain mostly multipotent hematopoietic precursors (Godin et al., 1995, 1999). They are capable of generating clones of lymphoid and myeloid lineages in vitro and of long-term hematopoietic reconstitution when injected intravenously into immunodeficient mice (Muller et al., 1994; Cumano et al., 2001). Here we exposed these embryonic HSCs to neural environments to examine whether they can differentiate into neural cells by coculturing them with multipotential neural precursors or by grafting them into the ventricles of newborn mice.

Because myelin mutants strongly stimulate grafted wild-type 
neural cells to myelinate, they seemed particularly appropriate hosts to compare the putative neural potential of HSCs with the well established myelinating potential of neural precursors (Vitry et al., 2001). We therefore transplanted purified and expanded hematopoietic AGM precursors into the brain ventricles of shiverer mice that are deficient in myelination because they lack myelin basic protein (MBP) (Vitry et al., 2001). Our results show that these AGM precursors integrate well in normal and dysmyelinating brain in which they develop into microglial cells, the brain macrophages. In contrast to neural precursors, primitive hematopoietic precursors do not generate neural cells in normal newborn brain or myelinating oligodendrocytes in shiverer brain. Thus, primitive AGM-HSCs give rise to microglia but not neurons or myelin-forming cells, despite exposure to neural environment signals.

\section{Materials and Methods}

AGM cell purification and expansion. Animal procedures were performed in compliance with the Pasteur Institute regulations. Wild-type C3H/ $\mathrm{HeN}$ female mice (Janvier, Le-Genest-Saint-Isle, France) were mated with hemizygote transgenic C57BL/6-actin-enhanced green fluorescent protein (EGFP) male mice (catalog \#003291; The Jackson Laboratory, Bar Harbor, ME). With vaginal plug observation day counted as $0.5 \mathrm{~d}$ post-coitum (dpc), 10.5-11.5 dpc F1 progenies were screened under UV light to detect GFP. Dissociated AGM cells were labeled with the following antibodies (all from BD Biosciences, Le-Pont-de-Claix, France): $R$-Phycoerythrin (PE)-conjugated anti-mouse CD45 (clone 30-F11), Allophycocyanine (APC)-conjugated anti-mouse CD44 (clone IM7), APCconjugated anti-CD117 or biotin-conjugated anti-mouse CD117 (ckit; clone 2B8), and biotin-conjugated anti-mouse AA4.1 (clone 493) followed by streptavidin Cy-Chrome (SavCy). Cells were then sorted with a MoFlo sorter equipped with Summit Software and collected in microplates with a CyCLONE dispenser robot. Sorted cells were seeded at clonal or low density ( $14 \pm 4$ cells $/ \mathrm{cm}^{2}$ in $25 \mathrm{~cm}^{2}$ flasks), on an irradiated monolayer of S17 stromal cells in optiMEM medium (Invitrogen, CergyPontoise, France) with 5\% fetal calf serum (FCS), KIT ligand (KITL), and interleukin 7 (IL7) supernatants ("hematopoietic conditions"). Cells were viewed with a Zeiss (Le-Pecq, France) Axiovert 135 inverted microscope equipped with an Axiocam camera.

Samples of AGM cells seeded at low density and expanded for $4-5 \mathrm{~d}$ were adhered $30 \mathrm{~min}$ onto glass coverslips coated with $1 \mathrm{mg} / \mathrm{ml}$ poly-Llysine (PLL) (Sigma, Saint-Quentin-Fallavier, France) for immunolabeling. Other samples were reseeded at clonal density, and, after 2 weeks, wells containing clones $(3 \pm 0.8 \%)$ were incubated with PE-conjugated anti-mouse CD19 (clone 1D3) and APC-conjugated anti-mouse CD11b (clone M1/70). Live cells were examined with a FACScalibur and analyzed with the Cell Quest software.

AGM precursor cultures and cocultures. Some freshly sorted AGM cells were cultured in the presence of $20 \mathrm{ng} / \mathrm{ml}$ human epidermal growth factor (EGF) (Sigma) and $10 \mathrm{ng} / \mathrm{ml}$ recombinant basic fibroblast growth factor (FGF2) (AbCys, Paris, France) supplied in (1) in optiMEM 5\% FCS with or without KITL and IL7, sometimes conditioned by S17 stromal cells or (2) DMEM-F-12 with B27 or N2 (Invitrogen), sometimes conditioned by mouse neurospheres (Calaora et al., 2001).

Expanded EGFP-positive $\left(\mathrm{EGFP}^{+}\right) \mathrm{AGM}$ cells were cocultured at a 1:3-1:4 ratio with dissociated striatal precursors (from wild-type $\mathrm{C} 3 \mathrm{H}$ / $\mathrm{HeN}$ mouse embryos) and grown in DMEM-F-12 supplemented with B27, EGF, and FGF2 ("neural conditions"). After 7 d, chimeric neurospheres were adhered onto $35 \mathrm{~mm}$ Petri dishes coated with $20 \mu \mathrm{g} / \mathrm{ml}$ PLL followed by $20 \mu \mathrm{g} / \mathrm{ml}$ Fibronectin or Laminin (Sigma). Adherent spheres grown for $7 \mathrm{~d}$ in DMEM-F-12-B27 and 1\% FCS were fixed with 2\% formaldehyde and immunolabeled.

Transplantation in newborn brain. We used wild-type $\mathrm{C} 3 \mathrm{H} / \mathrm{HeN}$ newborn mice and F1 from homozygote shiverer mice (catalog \#001428; The Jackson Laboratory), which do not show tremor at birth and were all devoid of MBP. Postnatal day 1 (P1) to P3 wild-type and shiverer pups were irradiated (200 Rad), cryoanesthetized, and injected with the fol- lowing: $4.10^{3}$ to $16.10^{3} \mathrm{EGFP}^{+} \mathrm{AGM}$ cells $(n=11), 4.10^{3} \mathrm{GFP}^{+} \mathrm{AGM}$ cells mixed with $12.10^{3}$ wild-type neurosphere cells $(n=3)$, and $12.10^{3}$ $\mathrm{GFP}^{+}$neurosphere cells $(n=3)$. Cell suspensions $(3 \mu \mathrm{l})$ were stereotaxically implanted into the left cerebral ventricle with a siliconized glass micropipette connected to a $10 \mu$ l Hamilton syringe (Phymep, Paris, France) and according to the following anteroposterior (AP), lateral (L), and vertical (V) coordinates in $\mathrm{mm}$ ): AP, $2 ; \mathrm{L}, 1 ; \mathrm{V}, 1$ for $\mathrm{P} 1$ recipients; and $\mathrm{AP}, 2.5$; L, $1.5 \mathrm{~mm}$; V, $1.5 \mathrm{~mm}$ for $\mathrm{P} 2$ and $\mathrm{P} 3$ recipients. After $1-3$ weeks, animals were anesthetized and perfused as described previously (Vitry et al., 2001). Host brains were postfixed $2 \mathrm{hr}$ before vibratome sections $(100 \mu \mathrm{m})$ or $1 \mathrm{hr}$ before cryoprotection overnight at $4^{\circ} \mathrm{C}$ in PBS $15 \%$ sucrose (Sigma) and cryostat sectioning $(12 \mu \mathrm{m})$.

Immunochemistry. Fixed cells or brain sections were treated with icecold ethanol for $5 \mathrm{~min}$ and incubated overnight at $4^{\circ} \mathrm{C}$ with the following antibodies: anti-GFP (Invitrogen), CD45 (rat IgG2b), CD11b, F4/80 (rat IgG2b; Serotec, Cergy-Saint-Christophe, France), nestin (mouse IgG1; BD Biosciences), polysialylated neural cell adhesion molecule (PSANCAM) (mouse IgM), BIII tubulin (mouse IgG2a; Eurogentec, Angers, France); or neuronal-specific nuclear protein (NeuN) (mouse IgG1; Chemicon, Temecula, CA), O4 (mouse IgM), 2' , 3' -cyclic nucleotide $3^{\prime}$ phosphodiesterase (CNP) (mouse IgG1; Sigma), and glial acidic fibrillary protein (GFAP) (mouse IgG1; Sigma), MBP [mouse IgG1 (Innogenetics, Lille, France) or rat IgG (Euromedex, Souffelweyersheim, France)]. Antibodies were diluted in $2 \%$ bovine serum albumin and $2 \%$ normal goat serum in PBS, with $0.05 \%$ Triton X-100 in the case of intracellular epitopes. The cells were then rinsed in PBS and incubated $1 \mathrm{hr}$ at room temperature with appropriate secondary antibodies and Hoechst 33342 $(5 \mu \mathrm{g} / \mathrm{ml})$ (Sigma). Coverslips were mounted in Fluoromount (Clinisciences, Montrouge, France), and sections were observed with a Leica (Reuil-Malmaison, France) DMRBE confocal microscope equipped with a TCS4D camera. The number of transplanted EGFP ${ }^{+}$cells and the double-labeled microglia were counted in a fraction of the brain sections in 11 grafted mice. Estimates of total numbers of engrafted cells were obtained by normalizing the counts to the total number of sections per brain.

\section{Results}

AGM cells are multipotent in vitro even after expansion

A purified primordial HSC population, which exhibits multipotentiality in vitro and long-term reconstitution capacity in vivo, can be obtained from $10.5 \mathrm{dpc}$ embryonic AGMs by labeling for the pan-hematopoietic marker CD45, the tyrosine kinase receptor $c k i t$, the homing cell adhesion molecule CD44, and the early B cell lineage marker AA4.1 (Godin et al., 1995; Sanchez et al., 1996). We used this approach to sort HSCs from the AGM of 10.5 dpc transgenic actin-EGFP mouse embryos (Fig. 1a). We selected $\mathrm{EGFP}^{+}$cells for the absence of CD45, which at this stage is expressed by myeloid precursors only, and the presence of ckit together with either CD44 or AA4.1. This purification results in a population that generates multipotent colonies at a frequency of 1:2-1:3 (J. Y. Bertrand and A. Cumano, personal communication). These sorted cells were then expanded onto an irradiated feeder layer of S17 stromal cells at clonal or low-density seeding in the presence of KITL and IL7 (Fig. 1b,c). Only after seeding at low density and expanding for 4-5 d did we obtain enough cells for our neural differentiation experiments (mean \pm SEM, $11.10^{3} \pm$ $4.10^{3}$ cells $/ \mathrm{cm}^{2}$ ) (Fig. 1c). To determine whether these expanded AGM populations seeded at low density still contain multipotent hematopoietic cells, we reseeded them at one cell per well in hematopoietic conditions for 2 weeks. We examined by flow cytometry wells containing hematopoietic colonies for the presence of $\mathrm{CD}_{19}{ }^{+}$B lymphoid and/or CD11b ${ }^{+}$(Mac1 or complement receptor) myeloid cells (Fig. 1d). Analysis of five independent experiments showed that $28.7 \pm 10.0 \%$ of the cells collected after in vitro expansion maintained their lympho-myeloid multipotentiality, whereas $23.3 \pm 8.3$ and $48.0 \pm 15.9 \%$ had become 
a
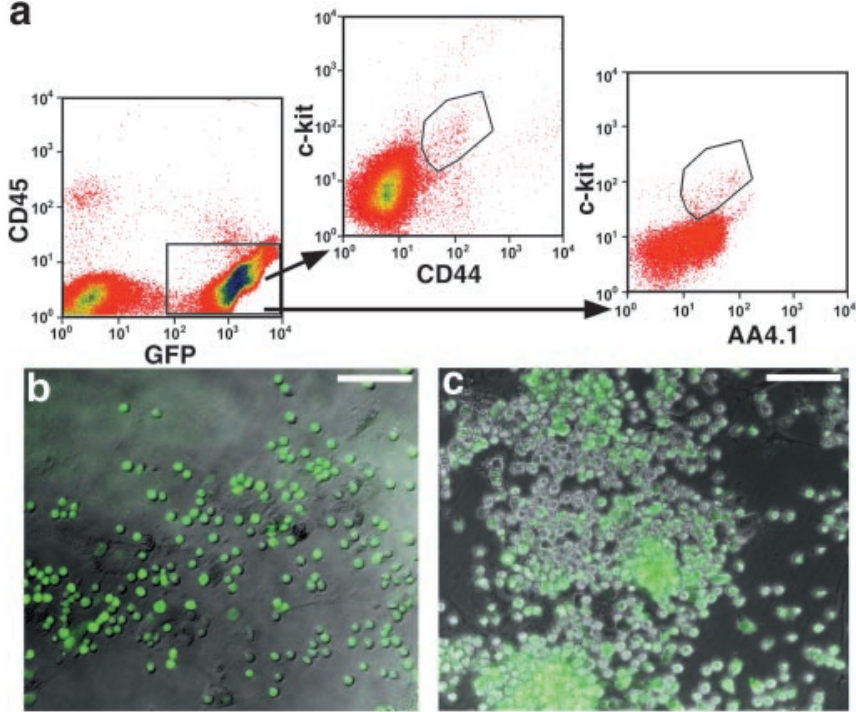

d
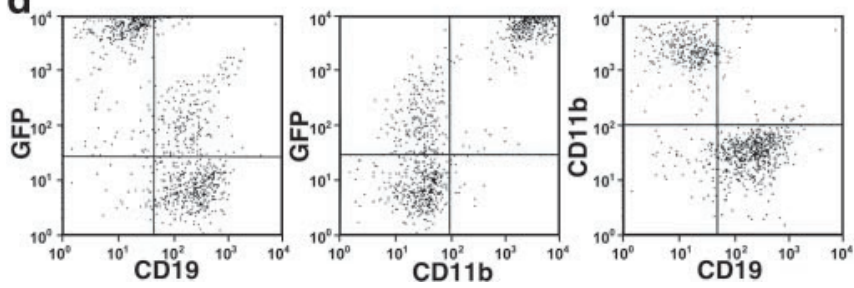

Figure 1. Selection and in vitro expansion of AGM-derived hematopoietic cells. $a$, FACS sorting of EGFP ${ }^{+}$AGM cells from 10.5-11.5 dpc actin-EGFP mice with no expression of CD45 (left) and high expression of ckit and CD44 (middle), or ckit and AA4.1 (right). b, Clonal expansion of AGM cells resulted in low cellular yields after $6 \mathrm{~d}$ in vitro. c, Expansion of AGM cells seeded at low density $\left(30\right.$ cells $/ \mathrm{cm}^{2}$ ) for $5 \mathrm{~d}$ provided much larger numbers of hematopoietic populations. Note the variable levels of EGFP expression in $b$ and $c . d$, FACS analysis of one representative hematopoietic clone obtained from cells initially expanded at low density. The left panel shows variable expression of EGFP in $\mathrm{CD} 19^{+}$lymphoid cells, whereas the middle panel demonstrates high expression of EGFP in most CD11b ${ }^{+}$myeloid cells. These two populations were derived from the same precursor and are shown together in the right panel (without the EGFP channel). Of the clones analyzed, $28.7 \pm 10 \%$ contained both $\mathrm{CD} 9^{+}$lymphoid and $\mathrm{CD} 11 \mathrm{~b}{ }^{+}$myeloid cells, indicating the persistence of multipotent cells after in vitro expansion. Scale bars: $b, c, 100 \mu \mathrm{m}$.

committed to the lymphoid and myeloid lineages, respectively. These results show that a fraction of sorted AGM precursors can maintain multipotentiality during the expansion period. We shall call these AGM hematopoietic stem cells "AGM-HSCs" throughout this manuscript.

\section{AGM-HSCs grown in hematopoietic conditions can express neural markers}

We then asked whether AGM-HSCs expanded in hematopoietic conditions express neural antigens (Fig. 2). Indeed, we found that a substantial proportion of round shaped $\mathrm{CD} 45^{+}$cells expressed the neural stem cell marker nestin $(24.6 \pm 2.1 \%)$ (Fig. $2 a)$, the neural plasticity marker PSA-NCAM $(20.8 \pm 4.8 \%)$ (Fig. $2 b$ ), the neuronal $\beta$ III tubulin protein $(21.6 \pm 1.3 \%$ ) (Fig. $2 c$ ), or the astrocytic GFAP marker (12.9 $\pm 1.7 \%$ ) (Fig. $2 d$ ). However, we did not find colabeling with the $\mathrm{O} 4$ antibody, which is specific for oligodendrocytes. Interestingly, when we attempted to grow AGM-HSCs in neural conditions with EGF and FGF2 with different conditioned media, we did not observe growth or survival of these hematopoietic precursors, suggesting that, despite the expression of some neural markers, they did not share the growth properties of neural precursors.
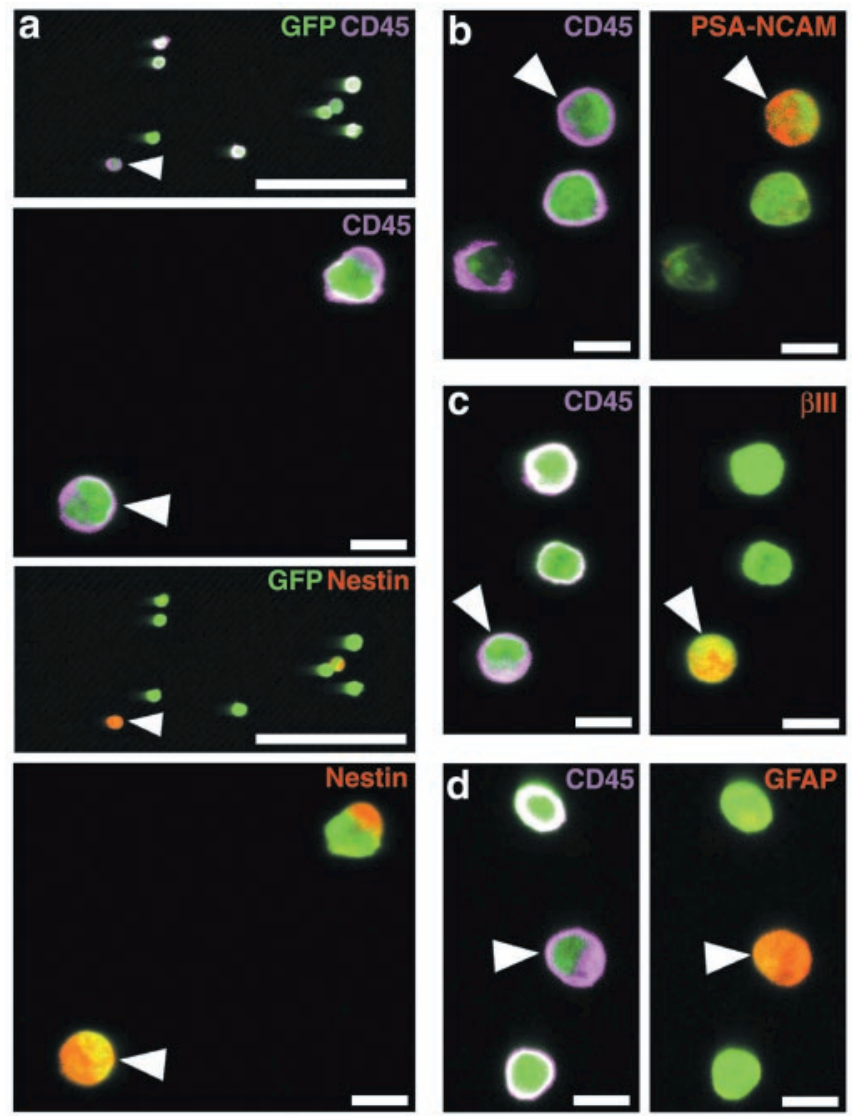

Figure 2. Expression of neural antigens in $\mathrm{CD} 45^{+} \mathrm{AGM}-\mathrm{HSCs}$. After growing for $4 \mathrm{~d}$ in hematopoietic conditions, many EGFP ${ }^{+}$AGM sorted precursors expressed CD45 protein (purple), and some of these coexpressed neural antigens (red). $a$, The top left panel shows a low-power view of cells expressing CD45 (arrowhead), and the underneath shows a higher-power confocal image of the surface ring-like localization of CD45 (appearing as purple or white by fusion of green and purple). The next left two panels below show low and high magnifications of $\mathrm{CD} 5^{+} /$nestin ${ }^{+}$cells (red at arrowhead) showing more staining at one pole of the cell. $b$ and c show confocal images of the colocalization of CD45 with PSA-NCAM staining (red at arrowhead in $b$ ) and $\beta \mathrm{III}$ tubulin (red at arrowhead in c) appearing polarized. In contrast, GFAP is diffuse in $\mathrm{CD}^{+}{ }^{+}$cells (red at arrowhead in $d$ ). Note that these CD45 cells, including those expressing neural antigens, invariably keep a round or oval shape. Scale bars: low-power views in $a, 100$ $\mu \mathrm{m}$; higher power views in $a-d, 10 \mu \mathrm{m}$.

\section{AGM-HSCs cocultured with neural precursors do not adopt a} neural fate

Because AGM-HSCs may require contact with neural cells to acquire neural potential, we cocultured EGFP ${ }^{+}$AGM cells with embryonic striatal precursors in the presence of EGF and FGF2. This led to formation of chimeric aggregates resembling neurospheres (Fig. 3a). When we adhered such neurospheres onto a substrate, cells migrated out and were immunolabelled for hematopoietic and neural markers. We found that $\mathrm{EGFP}^{+} \mathrm{AGM}-$ HSCs still expressed CD45 (Fig. 36 ) and were also positive for the myeloid CD11b marker (Fig. 3c) and the macrophage F4/80 antigen (Fig. 3d). Most of these cells displayed an amoeboid morphology or were occasionally elongated unipolar cells (Fig. 3b, inset, $f)$. Their close contacts with EGFP-negative $\left(\mathrm{EGFP}^{-}\right)$neural cells expressing nestin, GFAP, or $\beta$ III tubulin, or with oligodendrocytes labeled by the $\mathrm{O} 4$ antibody, did not trigger expression of neural markers in EGFP ${ }^{+}$AGM-derived cells (Fig. $3 b-e$ ). The only exception was for MBP, which was detected in some EGFP $^{+}$AGM cells (Fig. 3f, arrowheads). MBP-related transcripts indeed encode proteins expressed in cells of the hematopoietic lineage (Marty et al., 2002). 

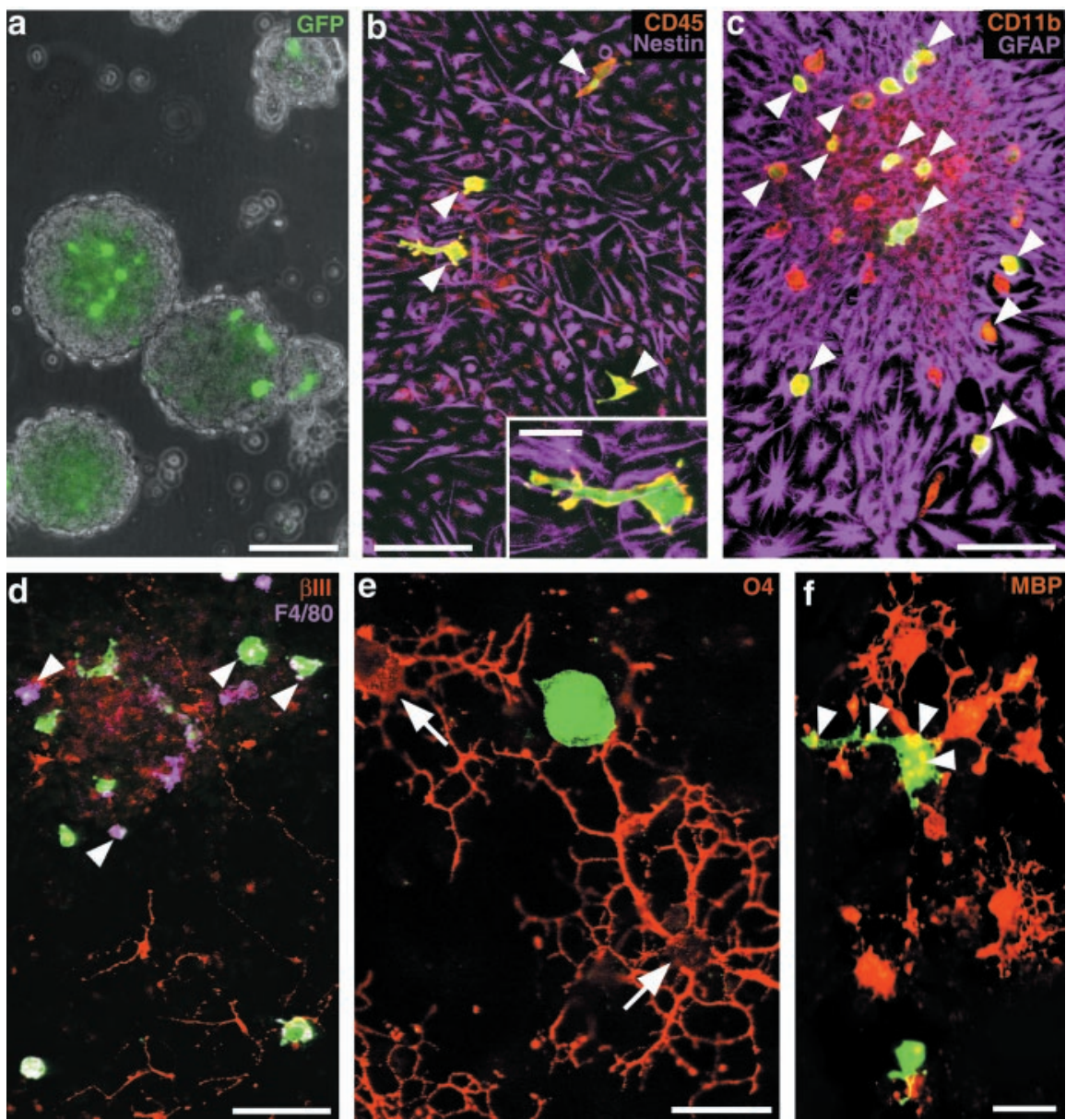

Figure 3. Coculture of EGFP ${ }^{+} \mathrm{AGM}-\mathrm{HSC}$ s with wild-type embryonic neural precursors. $a, \mathrm{EGFP}^{+} \mathrm{AGM}$ sorted cells mixed with embryonic striatal precursors have integrated in neurospheres after $7 \mathrm{~d}$ with $\mathrm{EGF}$ and FGF2. $b-f$, Seven days after adhesion of these chimeric neurospheres, cells that migrated in the outgrowth contained green EGFP ${ }^{+}$AGM cells that expressed CD45 (red, resulting in orange label at arrowheads in $b$ ) and were scattered among nestin ${ }^{+}$neural precursors (purple in $b$ ). In c, EGFP ${ }^{+}$ AGM-derived cells have differentiated in macrophages expressing CD11b (orange at arrowheads) among numerous GFAP ${ }^{+}$ purple astrocytes. $d, \beta$ III tubulin ${ }^{+}$neuronal cells with processes (red) did not express EGFP, whereas other round EGFP ${ }^{+}$cells expressed F4/80 (purple at arrowheads). e, Two 04 antibody-labeled oligodendrocytes (red at arrows) showed typical multiple

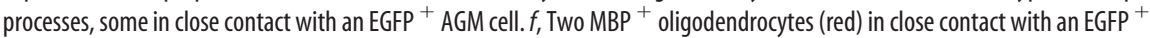
AGM cell that shows some orange dots (at arrowheads). Except in this case, all EGFP ${ }^{+}$AGM sorted cells shown in $b-d$ only expressed hematopoietic markers (CD45, CD11b, or F4/80). $b$-fare confocal images. Scale bars: $a-d, 100 \mu \mathrm{m}$; inset in $b, e, f, 25$ $\mu \mathrm{m}$.

\section{AGM-HSCs engraft the developing brain and generate microglia}

To determine whether primordial HSCs can engraft the CNS, we transplanted expanded EGFP ${ }^{+}$AGM-HSCs into the developing brain of irradiated wild-type and shiverer newborn mice. After 6-8 d, both normal and mutant host brains $(n=5$ of 5$)$ contained donor-derived cells around the cerebral ventricles, in the choroid plexus, the corpus callosum, the hippocampus, and/or the striatum (Figs. 4, 5). The ratio of cells engrafted at this time reached $74 \pm 20 \%$. We also found AGM cells at $17-21 \mathrm{~d}$ after graft, although in smaller numbers ( $n=7$ of $9 ; 21 \pm 6 \%$ engrafted cells). In all cases, the vast majority of grafted AGM-HSCs evolved into cells expressing the macrophage $\mathrm{F} 4 / 80$ antigen ( $85 \pm$ $3 \%$ at 1 week and $90 \pm 2 \%$ at 3 weeks) and showed a characteristic phenotype of ramified microglia (Fig. 4), which was more frequent than the amoeboid phenotype. Some round or amoeboid $\mathrm{EGFP}^{+}$cells that expressed CD11b or CD45 were lining the third ventricle or were integrated into the choroid plexus (Fig. 5a,b). At both time points examined, none of the grafted cells developed into neurons expressing the neuron nuclear protein NeuN or astrocytes, even when in close contact with these cells (Fig. $5 c, d)$, or into oligodendrocytes when intermingled with myelinated fibers in wildtype mice (Fig. 5e).We conclude from these results that AGM-HSCs integrated well in neonatal brain and differentiated into microglia.

\section{AGM-HSCs do not generate myelin forming cells in shiverer brain} Because the dysmyelinated shiverer environment stimulates myelination by grafted neural precursors (Vitry et al., 2001), shiverer hosts grafted with EGFP ${ }^{+}$ AGM-HSCs were compared with those transplanted with $\mathrm{EGFP}^{+}$neural precursors. We analyzed expression of two myelin proteins: CNP, expressed by both grafted and shiverer oligodendrocytes; and MBP, restricted to donor-derived wildtype oligodendrocytes and their myelin. Shiverer mice indeed can make thin $\mathrm{CNP}^{+}$ myelin internodes whose presence is an indication of myelination progress. Eight days after grafting, rare $\mathrm{EGFP}^{+} \mathrm{AGM}$ cells were found to express MBP as observed in vitro. However, these $\mathrm{MBP}^{+}$cells did not display the typical morphology of myelinating cells (Fig. $6 a$ ). In contrast, EGFP ${ }^{+}$ neural precursors generated $\mathrm{MBP}^{+}$oligodendrocytes with numerous processes (Fig. 6d).

We then examined shiverer mice grafted with $\mathrm{EGFP}^{+}$AGM-HSCs at $17 \mathrm{~d}$, at the time of active myelination by $\mathrm{CNP}^{+}$ oligodendrocytes but found no $\mathrm{EGFP}^{+} /$ $\mathrm{MBP}^{+}$myelinating cells (Fig. 6b). However, in shiverer brains grafted with $\mathrm{GFP}^{+}$neural precursors and examined at the same time, well developed $\mathrm{MBP}^{+}$myelin patches were closely associated with $\mathrm{EGFP}^{+}$oligodendrocyte cell bodies (Fig. 6e).

To verify that the different fate of AGM-HSCs (compared with that of neural precursors) was not attributable to variation in environmental signals from one shiverer recipient to another, we cotransplanted these EGFP ${ }^{+}$AGM cells with wild-type neural precursors. After $17 \mathrm{~d}$, wild-type $\mathrm{EGFP}^{-} / \mathrm{MBP}^{+}$donor cells extended fine processes to $\mathrm{MBP}^{+}$myelin internodes, whereas EGFP $^{+}$AGM cells in the vicinity exhibited microglial morphology (Fig. 6f) or stayed round and apparently unable to extend processes around host axons and form myelin (Fig. $6 g$ ). Together, these results indicate that AGM-HSCs, in contrast to neural precursors, do not develop a myelinating potential in this permissive environment.

\section{Discussion}

In this study, we explored for the first time the transdifferentiation potential of a well characterized population of primordial HSCs, AGM-HSCs, when exposed to neural environments in vitro and in vivo. We show that expanded AGM-HSCs precursors derived from $\mathrm{EGFP}^{+}$mice contain close to $30 \%$ multipotent cells 

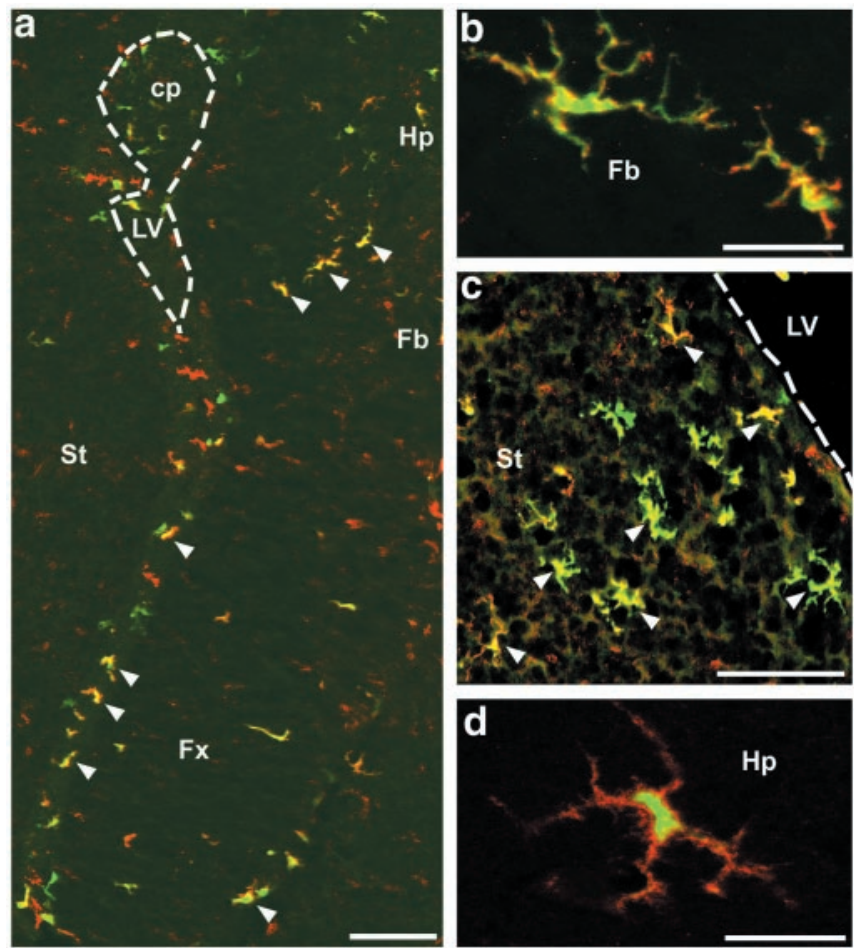

Figure 4. $\mathrm{EGFP}^{+} \mathrm{AGM}-\mathrm{HSC}(\mathrm{s}$ grafted into newborn brain become microglia. In both shiverer $(a-c)$ and wild-type (d) mice, AGM donor cells evolved into F4/80-expressing microglia with typical long branched processes. Eight days ( $a$ and $b$ ) and $17 \mathrm{~d}(c)$ after grafting into shiverer brain, some donor cells were integrated in areas lining the lateral ventricle (delineated by dotted line) and the striatum. Several green donor cells were double labeled by the antibody $\mathrm{F} 4 / 80$ (in red) along their processes (orange at arrowheads). The detailed morphology of ramified microglia derived from AGM donor cells is identical after grafting AGM-HSCs in shiverer ( $c$ ) or wild-type ( $d$ ) brain. $\mathrm{cp}$, Choroid plexus; Fb, fimbriae; Fx, fornix; Hp, hippocampus; LV, lateral ventricle; St, striatum. Scale bars: $a, c, 100 \mu \mathrm{m} ; b, d, 25 \mu \mathrm{m}$.

after 4-5 d in vitro. AGM-HSCs cocultured with growing neurospheres continue to display hematopoietic markers and do not become neural cells. Instead, both in vitro and after grafting into newborn wild-type or shiverer mice, they give rise to cells of the macrophage/microglia lineage. Similarly, adult BM cells grafted in immunodeficient animals generated many microglial cells (Eglitis and Mezey, 1997). Even after cografting neural precursors and $\mathrm{EGFP}^{+}$HSCs in the MBP-deficient mouse brain, each type of precursor adopted its expected fate, microglia in the case of AGM-HSCs and myelin-forming cells in the case of neural precursors. Thus, we found no evidence that primordial HSCs respond to neonatal and hypomyelinated brain signals by transdifferentiation into myelinating cells.

Expression of "neural" antigens in cells not derived from neuroectoderm does not necessarily correlate with a potential to become neural cells (Temple, 2001). In fact, a subpopulation of AGM-HSCs that expressed some of these antigens when grown in hematopoietic conditions did not develop into neurons or glia when confronted with neural environments in vitro and in vivo. Interestingly, some of the AGM-HSCs evolving into microglia started to express MBP in vitro and in vivo. The MBP gene is composed of two transcriptional units. One of these starts upstream of the classical MBP start sites and gives rise to the "Golli" transcripts, which are not only expressed in brain but also in lymphoid and myeloid lineages, including macrophages (Pribyl et al., 1993; Marty et al., 2002). The monoclonal MBP antibody used here likely recognizes an epitope in the protein encoded by
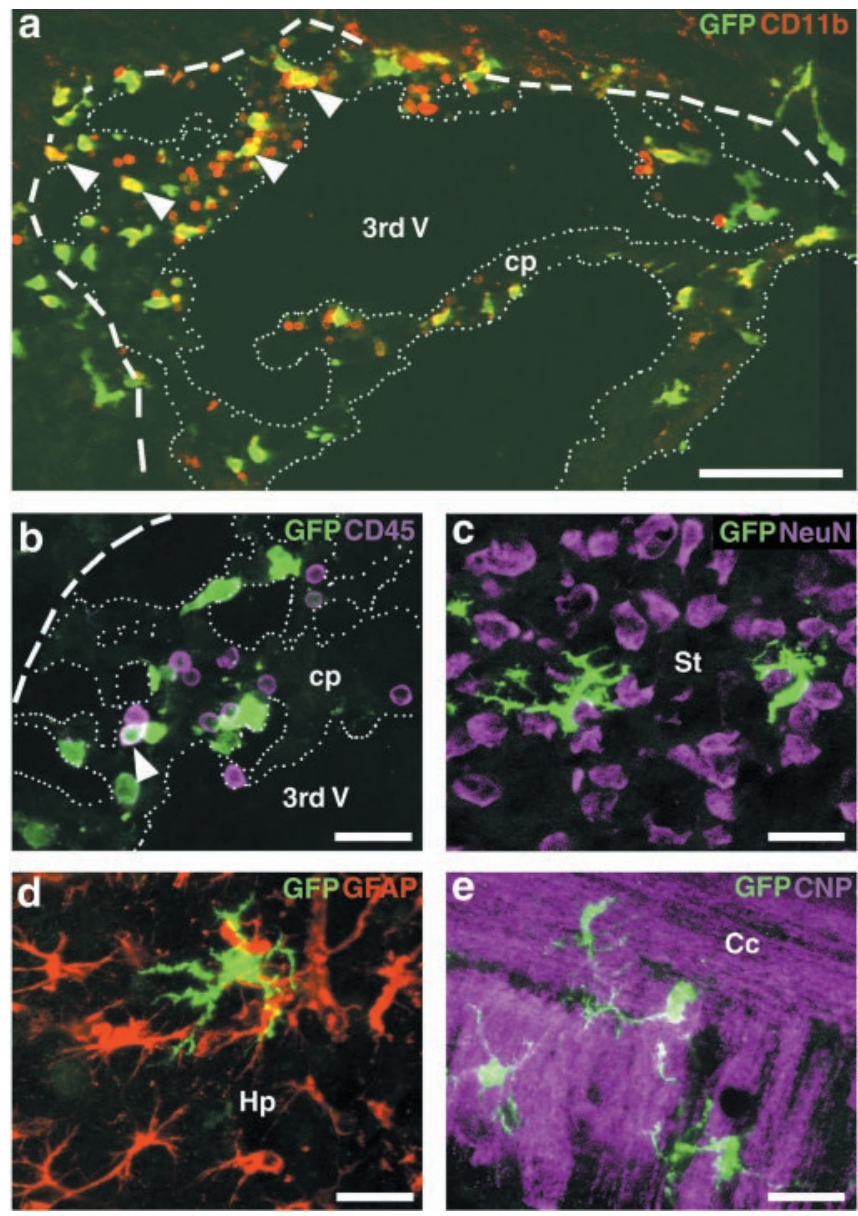

Figure 5. $\mathrm{EGFP}^{+} \mathrm{AGM}-\mathrm{HSC}$ engrafted in brain do not express neural markers. $a, b$, Eight days after transplantation into shiverer brain, the choroid plexus (thin dotted line) located in the third ventricle (thick dotted line) contained many EGFP ${ }^{+} \mathrm{AGM}$ donor cells with round or amoeboid shape, some expressing the CD11b myeloid marker (arrowheads in $a$ ). CD45 at the surface of round cells (purple in $b$ ) also labels one EGFP ${ }^{+}$donor cell (arrowhead in $b$ ). $c-e$, Seventeen to $21 \mathrm{~d}$ after graft, EGFP ${ }^{+}$ramified microglial cells have integrated the shiverer striatum $(c)$, as well as the wild-type mice hippocampus ( $d$ ) and corpus callosum (e). EGFP ${ }^{+}$microglial cells intermingled with neural cells expressing NeuN (purple in c) or GFAP (red in $d$ ) or with CNP ${ }^{+}$ myelinated fibers (purple in e), but none of these green donor-derived cells coexpressed neural antigens. cp, Choroid plexus; Cc, corpus callosum; Hp, hippocampus; St, striatum; 3rd V, third ventricle. Scale bars: $a, 100 \mu \mathrm{m} ; b-e, 25 \mu \mathrm{m}$.

one of the Golli transcript. Thus, it is conceivable that the MBPpositive fraction of $\mathrm{CD} 45^{+}$cells did not express MBP from classical MBP transcripts and may thus not belong to the oligodendrocyte lineage, in contrast to $\mathrm{MBP}^{+}$cells derived from neural precursors that gave rise to authentic, myelinating oligodendrocytes.

Previous studies have explored the plasticity and myelinating potential of adult BM populations. Grafted BM populations enriched in ckit-positive precursors integrated well in newborn brain after grafting and generated cells expressing preoligodendrocytic, astrocytic, and neuronal markers (Bonilla et al., 2002). Adult BM cells grafted in a focal lesion or delivered intravenously generated myelinating Schwann cells, and this resulted in enhanced conduction velocity in remyelinated axons (Sasaki et al., 2001; Akiyama et al., 2002a). Schwann cells normally myelinate peripheral nerves but can also remyelinate CNS axons. When BM-derived adherent cells expressing stromal cell markers were grafted in the same model, axons became remyelinated (Akiyama et al., 2002b). Although these studies offer exciting prospects, particularly if autologous stroma cells from BM aspirates could 

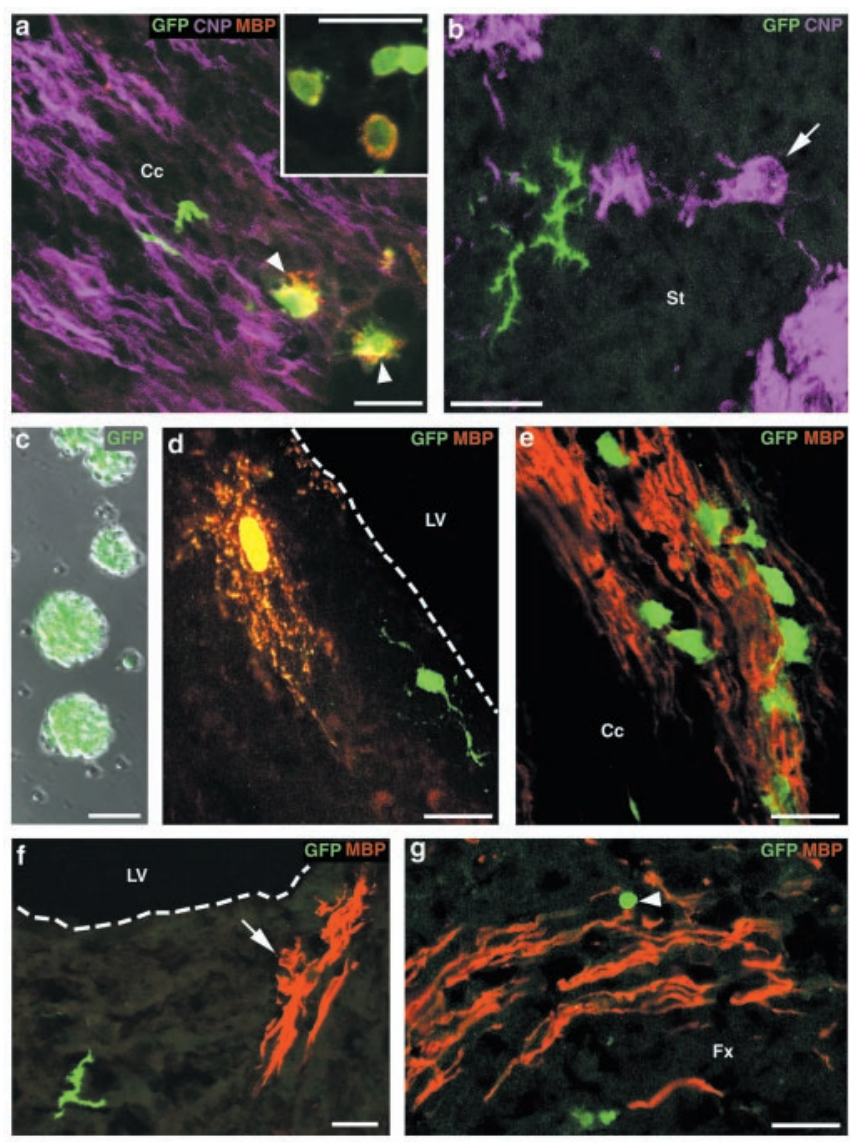

Figure 6. Neural precursors but not AGM-HSCs can myelinate shiverer brain. $a$, One week after graft, some EGFP ${ }^{+}$AGM cells were found in white matter in which thinly myelinated fibers were detected by CNP immunolabelling (purple). Some of these cells expressed low levels of MBP in fine red processes (arrowheads). MBP was also found in a round EGFP ${ }^{+}$AGM cell located at the border of the third ventricle (inset in $a$ ). $b$, Seventeen days after graft, no MBPexpressing cells were found among microglia derived from AGM cells even in brain regions harboring CNP ${ }^{+} / \mathrm{MBP}^{-}$shiverer host myelinating oligodendrocytes (purple at arrow). $c-e$, $\mathrm{EGFP}^{+}$striatal neurophere-derived cells ( $c$ ) were transplanted into shiverer. After 1 week, an $\mathrm{EGFP}^{+}$neural precursor has evolved into a premyelinating oligodendrocyte expressing MBP in its cell body and its multiple long processes (orange in $d$ ). A bipolar EGFP ${ }^{+}$cell on the right may correspond to a putative oligodendrocyte progenitor that does not yet express MBP. e, Seventeen days after graft, EGFP ${ }^{+}$oligodendrocyte cell bodies are seen in association with MBP ${ }^{+}$ myelin internodes. Note that there is no more MBP in the cell body at that time because this protein has moved to the myelinating internodes. $f, g$, Cografting of EGFP ${ }^{+}$AGM cells with wild-type GFP-negative neurosphere cells resulted in engrafment of both cell populations after $17 \mathrm{~d}$. Green ramified or round EGFP ${ }^{+}$AGM cells were found around $(f)$ or in close association with (at arrowhead in $g$ ) MBP ${ }^{+}$myelin (red) synthesized by GFP-negative oligodendrocytes derived from wild-type neural precursors. Arrow in $f$ indicates one $\mathrm{MBP}^{+}{ }^{+}$oligodendrocyte cell body connected to its myelin internodes by two thin processes. (c, Corpus callosum; Fx, fornix $\mathrm{LV}$, lateral ventricle; St, striatum. Scale bars: $a, b, d-g, 25 \mu \mathrm{m} ; c, 100 \mu \mathrm{m}$.

be expanded and delivered peripherally, it is important to determine whether the grafted cells indeed became the remyelinating cells or whether they promoted an endogenous repair process (Akiyama et al., 2002b).

Different mechanisms may underlie what has been called "transdifferentiation." First, the BM could be the source of multipotent-totipotent stem cells that may circulate in the blood and home in various organs, including the brain. In favor of this idea is the isolation from human fetal brain of stem cells expressing CD133, a marker for HSC (Uchida et al., 2000). Moreover, BMderived multipotent adult precursor cells can contribute to several mouse lineages, including muscle and nervous system (Jiang et al., 2002). HSC ability to home in niches in various organs may result in isolation of discrete subsets of HSC present in one particular organ. Homing of HSC is controlled by the chemokine receptor CXCR4, which binds the $\alpha$ chemokine CXCL12 expressed at high concentration in these niches (PituchNoworolska et al., 2003). CXCL12 is synthesized in the CNS early in the embryo and throughout life (Lazarini et al., 2003), reinforcing the proposal that CXCL12 niches may exist in the brain. Second, BM cells can adopt other cell fate (such as chondrocytes or striated muscle cells) by cell fusion (Terada et al., 2002). Similarly, mouse CNS cells cocultured with embryonic stem cells can form tetraploid hybrids harboring multipotency (Ying et al., 2002). This event might be rare in vivo, however, because diploid cells would have a selective advantage over tetraploid ones (McKay, 2002). Nevertheless, fusion between hepatocytes and grafted BM cells appears to be the major mechanism of experimental liver regeneration previously interpreted as the result of transdifferentiation (Lagasse et al., 2000; Wang et al., 2003). In our study, no evidence of fusion between AGM-HSCs and neural cells was detected.

Experiments performed in immunodeficient animals have shown that unselected BM-derived cells can integrate into the brain and generate microglia, neurons, and astrocytes (Brazelton et al., 2000; Mezey and Chandross, 2000). The exact origin of these neural cells remains unknown because total BM cells contain only $0.05-0.5 \%$ HSCs and $\sim 0.125 \%$ stromal cells (Mezey and Chandross, 2000). The frequency of neurons generated by these grafted BM cells has been estimated to be $0.3-2.3 \%$, but this may occur more rarely in female patients having undergone BMT from male donors (Mezey et al., 2003; Weimann et al., 2003). The reproducibility and/or frequency of these events in immunosuppressed mice have been challenged by other studies in which BM-derived neural cells were not observed. However, donor cells that survived in the brain for several months maintained expression of hematopoietic markers (Castro et al., 2002; Ono et al., 2003; Vallieres and Sawchenko, 2003). In similar experiments, neogenesis of Purkinje cells in cerebellum has been described as a rare event (Priller et al., 2001), even when efficient reconstitution of the hematopoietic system was obtained with a single donor $\mathrm{GFP}^{+}$HSC (Wagers et al., 2002). Together, these studies indicate that a robust contribution of BM cells and/or HSCs to an unexpected tissue was not observed, raising the possibility that the plasticity of such precursors is limited (Lemischka, 2002). Meanwhile, recent studies have suggested that umbilical cord blood contains precursors able to generate neural cells from adherent cells in vitro, including oligodendrocytes (Buzanska et al., 2002).

Contribution of grafted BM cells or stem cells to brain repair could be more substantial in neurological diseases, especially when the blood-brain barrier is altered. One remarkable observation is the therapeutic effect of BMT in early stage of adrenoleukodystrophy in which the mutation hits an intracellular transmembrane peroxisomal transporter expressed in oligodendrocytes, astrocytes, and microglia (Dubois-Dalcq et al., 1999). This suggests that mutated CNS cells were partially replaced by wild-type donor cells in these BMT recipient children, but it is unknown whether only the diseased microglia were replaced or some oligodendrocytes were also generated. Equally intriguing is the observation that BMT can stabilize some patients affected with progressive multiple sclerosis (Muraro et al., 2003). As predicted, the major effect of BMT in this case is a dramatic reduction of inflammation in the lesions, but a contribution of neural cell replacement to clinical stabilization cannot be excluded. An exciting new finding is that intravenous delivery of neurospheres can induce clinical recovery in an experimental model of multiple scle- 
rosis (Pluchino et al., 2003). Eventually, one should be able to trigger the mitosis and migration toward demyelinating lesions of endogenous adult neural precursors that have been characterized recently in rodent and man (Doetsch et al., 2002; Nunes et al., 2003). Indeed, EGF and FGF2 delivered intraventricularly in the mouse brain induced mitosis of subventricular zone precursors and, in the case of EGF, extensive migration in the brain parenchyma (Doetsch et al., 2002; Martens et al., 2002).

In conclusion, our study provides compelling evidence that AGM-HSCs are precursors able to generate microglial cells when exposed to neural environments, a property that was not known to be acquired so early in embryonic development. It also provides a detailed comparative analysis of the myelinating potential of two well characterized multipotential precursors, embryonic hematopoietic stem cells and striatal neural precursors grown into neurospheres. The latter remain the most potent source of precursors capable of oligodendrocyte and myelin regeneration in a myelin mutant, as demonstrated by transplantation (Mitome et al., 2001), and thus would seem to represent so far one of the most promising avenues toward remyelination therapy.

\section{References}

Akiyama Y, Radtke C, Honmou O, Kocsis JD (2002a) Remyelination of the spinal cord following intravenous delivery of bone marrow cells. Glia 39:229-236.

Akiyama Y, Radtke C, Kocsis JD (2002b) Remyelination of the rat spinal cord by transplantation of identified bone marrow stromal cells. J Neurosci 22:6623-6630.

Bjornson CR, Rietze RL, Reynolds BA, Magli MC, Vescovi AL (1999) Turning brain into blood: a hematopoietic fate adopted by adult neural stem cells in vivo. Science 283:534-537.

Bonilla S, Alarcon P, Villaverde R, Aparicio P, Silva A, Martinez S (2002) Haematopoietic progenitor cells from adult bone marrow differentiate into cells that express oligodendroglial antigens in the neonatal mouse brain. Eur J Neurosci 15:575-582.

Brazelton TR, Rossi FM, Keshet GI, Blau HM (2000) From marrow to brain: expression of neuronal phenotypes in adult mice. Science 290:1775-1779.

Buzanska L, Machaj EK, Zablocka B, Pojda Z, Domanska-Janik K (2002) Human cord blood-derived cells attain neuronal and glial features in vitro. J Cell Sci 115:2131-2138.

Calaora V, Rogister B, Bismuth K, Murray K, Brandt H, Leprince P, Marchionni M, Dubois-Dalcq M (2001) Neuregulin signaling regulates neural precursor growth and the generation of oligodendrocytes in vitro. J Neurosci 21:4740-4751.

Castro RF, Jackson KA, Goodell MA, Robertson CS, Liu H, Shine HD (2002) Failure of bone marrow cells to transdifferentiate into neural cells in vivo. Science 297:1299.

Cumano A, Ferraz JC, Klaine M, Di Santo JP, Godin I (2001) Intraembryonic, but not yolk sac hematopoietic precursors, isolated before circulation, provide long-term multilineage reconstitution. Immunity 15:477-485.

Doetsch F, Petreanu L, Caille I, Garcia-Verdugo JM, Alvarez-Buylla A (2002) EGF converts transit-amplifying neurogenic precursors in the adult brain into multipotent stem cells. Neuron 36:1021-1034.

Dubois-Dalcq M, Feigenbaum V, Aubourg P (1999) The neurobiology of $\mathrm{X}$-linked adrenoleukodystrophy, a demyelinating peroxisomal disorder. Trends Neurosci 22:4-12.

Eglitis MA, Mezey E (1997) Hematopoietic cells differentiate into both microglia and macroglia in the brains of adult mice. Proc Natl Acad Sci USA 94:4080-4085.

Godin I, Dieterlen-Lievre F, Cumano A (1995) Emergence of multipotent hemopoietic cells in the yolk sac and paraaortic splanchnopleura in mouse embryos, beginning at 8.5 days postcoitus. Proc Natl Acad Sci USA 92:773-777.

Godin I, Garcia-Porrero JA, Dieterlen-Lievre F, Cumano A (1999) Stem cell emergence and hemopoietic activity are incompatible in mouse intraembryonic sites. J Exp Med 190:43-52.

Jiang Y, Jahagirdar BN, Reinhardt RL, Schwartz RE, Keene CD, OrtizGonzalez XR, Reyes M, Lenvik T, Lund T, Blackstad M, Du J, Aldrich S,
Lisberg A, Low WC, Largaespada DA, Verfaillie CM (2002) Pluripotency of mesenchymal stem cells derived from adult marrow. Nature 418:41-49.

Lagasse E, Connors H, Al-Dhalimy M, Reitsma M, Dohse M, Osborne L, Wang X, Finegold M, Weissman IL, Grompe M (2000) Purified hematopoietic stem cells can differentiate into hepatocytes in vivo. Nat Med 6:1229-1234

Lazarini F, Tham TN, Casanova P, Arenzana-Seisdedos F, Dubois-Dalcq M (2003) Role of the alpha-chemokine stromal cell-derived factor (SDF-1) in the developing and mature central nervous system. Glia 42:139-148.

Lemischka I (2002) A few thoughts about the plasticity of stem cells. Exp Hematol 30:848-852.

Martens DJ, Seaberg RM, van der Kooy D (2002) In vivo infusions of exogenous growth factors into the fourth ventricle of the adult mouse brain increase the proliferation of neural progenitors around the fourth ventricle and the central canal of the spinal cord. Eur J Neurosci 16:1045-1057.

Marty MC, Alliot F, Rutin J, Fritz R, Trisler D, Pessac B (2002) The myelin basic protein gene is expressed in differentiated blood cell lineages and in hemopoietic progenitors. Proc Natl Acad Sci USA 99:8856-8861.

McKay R (2002) A more astonishing hypothesis. Nat Biotechnol 20:426-427.

Meletis K, Frisen J (2003) Blood on the tracks: a simple twist of fate? Trends Neurosci 26:292-296.

Mezey E, Chandross KJ (2000) Bone marrow: a possible alternative source of cells in the adult nervous system. Eur J Pharmacol 405:297-302.

Mezey E, Key S, Vogelsang G, Szalayova I, Lange GD, Crain B (2003) Transplanted bone marrow generates new neurons in human brains. Proc Natl Acad Sci USA 100:1364-1369.

Mitome M, Low HP, van den Pol A, Nunnari JJ, Wolf MK, Billings-Gagliardi S, Schwartz WJ (2001) Towards the reconstruction of central nervous system white matter using neural precursor cells. Brain 124:2147-2161.

Muller AM, Medvinsky A, Strouboulis J, Grosveld F, Dzierzak E (1994) Development of hematopoietic stem cell activity in the mouse embryo. Immunity 1:291-301.

Muraro PA, Ingoni RC, Martin R (2003) Hematopoietic stem cell transplantation for multiple sclerosis: current status and future challenges. Curr Opin Neurol 16:299-305.

Nunes MC, Roy NS, Keyoung HM, Goodman RR, McKhann II G, Jiang L, Kang J, Nedergaard M, Goldman SA (2003) Identification and isolation of multipotential neural progenitor cells from the subcortical white matter of the adult human brain. Nat Med 9:439-447.

Ono K, Yoshihara K, Suzuki H, Tanaka KF, Takii T, Onozaki K, Sawada M (2003) Preservation of hematopoietic properties in transplanted bone marrow cells in the brain. J Neurosci Res 72:503-507.

Pituch-Noworolska A, Majka M, Janowska-Wieczorek A, Baj-Krzyworzeka M, Urbanowicz B, Malec E, Ratajczak MZ (2003) Circulating CXCR4positive stem/progenitor cells compete for SDF-1-positive niches in bone marrow, muscle and neural tissues: an alternative hypothesis to stem cell plasticity. Folia Histochem Cytobiol 41:13-21.

Pluchino S, Quattrini A, Brambilla E, Gritti A, Salani G, Dina G, Galli R, Del Carro U, Amadio S, Bergami A, Furlan R, Comi G, Vescovi AL, Martino G (2003) Injection of adult neurospheres induces recovery in a chronic model of multiple sclerosis. Nature 422:688-694.

Pribyl TM, Campagnoni CW, Kampf K, Kashima T, Handley VW, McMahon J, Campagnoni AT (1993) The human myelin basic protein gene is included within a 179-kilobase transcription unit: expression in the immune and central nervous systems. Proc Natl Acad Sci USA 90:10695-10699.

Priller J, Persons DA, Klett FF, Kempermann G, Kreutzberg GW, Dirnagl U (2001) Neogenesis of cerebellar Purkinje neurons from gene-marked bone marrow cells in vivo. J Cell Biol 155:733-738.

Sanchez MJ, Holmes A, Miles C, Dzierzak E (1996) Characterization of the first definitive hematopoietic stem cells in the AGM and liver of the mouse embryo. Immunity 5:513-525.

Sasaki M, Honmou O, Akiyama Y, Uede T, Hashi K, Kocsis JD (2001) Transplantation of an acutely isolated bone marrow fraction repairs demyelinated adult rat spinal cord axons. Glia 35:26-34.

Shapiro E, Krivit W, Lockman L, Jambaque I, Peters C, Cowan M, Harris R, Blanche S, Bordigoni P, Loes D, Ziegler R, Crittenden M, Ris D, Berg B, Cox C, Moser H, Fischer A, Aubourg P (2000) Long-term effect of bonemarrow transplantation for childhood-onset cerebral X-linked adrenoleukodystrophy. Lancet 356:713-718. 
Temple S (2001) Stem cell plasticity: building the brain of our dreams. Nat Rev Neurosci 2:513-520.

Terada N, Hamazaki T, Oka M, Hoki M, Mastalerz DM, Nakano Y, Meyer EM, Morel L, Petersen BE, Scott EW (2002) Bone marrow cells adopt the phenotype of other cells by spontaneous cell fusion. Nature 416:542-545.

Uchida N, Buck DW, He D, Reitsma MJ, Masek M, Phan TV, Tsukamoto AS, Gage FH, Weissman IL (2000) Direct isolation of human central nervous system stem cells. Proc Natl Acad Sci USA 97:14720-14725.

Vallieres L, Sawchenko PE (2003) Bone marrow-derived cells that populate the adult mouse brain preserve their hematopoietic identity. J Neurosci 23:5197-5207.

Vitry S, Avellana-Adalid V, Lachapelle F, Evercooren AB (2001) Migration and multipotentiality of PSA-NCAM+ neural precursors transplanted in the developing brain. Mol Cell Neurosci 17:983-1000.
Wagers AJ, Sherwood RI, Christensen JL, Weissman IL (2002) Little evidence for developmental plasticity of adult hematopoietic stem cells. Science 297:2256-2259.

Wang X, Willenbring H, Akkari Y, Torimaru Y, Foster M, Al-Dhalimy M, Lagasse E, Finegold M, Olson S, Grompe M (2003) Cell fusion is the principal source of bone-marrow-derived hepatocytes. Nature 422:897-901.

Weimann JM, Charlton CA, Brazelton TR, Hackman RC, Blau HM (2003) Contribution of transplanted bone marrow cells to Purkinje neurons in human adult brains. Proc Natl Acad Sci USA 100:2088-2093.

Weissman IL, Anderson DJ, Gage F (2001) Stem and progenitor cells: origins, phenotypes, lineage commitments, and transdifferentiations. Annu Rev Cell Dev Biol 17:387-403.

Ying QL, Nichols J, Evans EP, Smith AG (2002) Changing potency by spontaneous fusion. Nature 416:545-548. 